\title{
Tough on germs, gentle on equipment
}

mikrozid universal from schülke UK experts in infection prevention - has a unique low alcohol formulation, with a dual action. Offering both cleaning and disinfection of surfaces in a single product, mikrozid universal is ideal where short contact times are required and prolonged exposure to moisture could cause damage. Effective against bacteria including $\mathrm{TB}$, and viruses like norovirus, mikrozid universal is compatible with a wide range of materials including keyboards, touch screens, leatherette furniture, surfaces of medical equipment and work surfaces.

mikrozid universal is fast acting (bactericidal in 15 seconds), has excellent material compatibility and contains added surfactants to boost cleaning performance. It is available in both liquid with integrated spray and high quality fleece wipes for enhanced coverage.

To find out more about how schülke's extensive infection prevention and control range can help your practice, visit www. schuelke.com or email: mail.uk@schuelke.com.

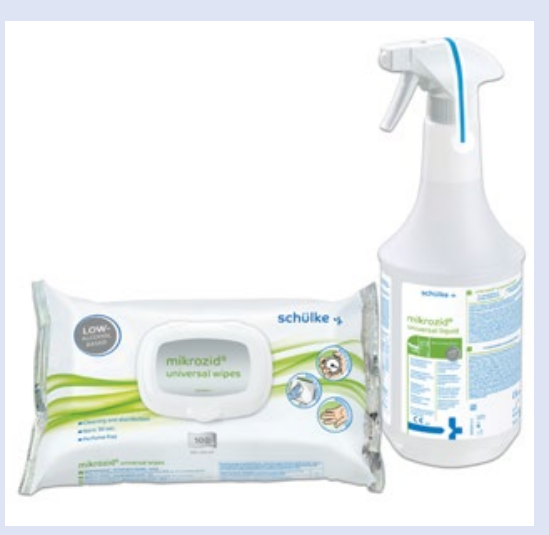

\section{Thinking differently about dentistry}

The all-new, two-day Dental Innovation Symposium, which took place on 7-8 June 2019 at the contemporary Pullman London St Pancras, was a huge success, with the inaugural event setting the world of dentistry on fire.

The symposium brought together a firstclass line-up of dentistry's leading experts in the area of business performance, as well as key opinion leaders from the world of digital and clinical dentistry. It was heralded as THE event for practices open to thinking differently about how to boost their business and meet patients' needs. Organisers Henry Schein Dental, Software of Excellence, BioHorizons and MediEstates carefully interwove the topics, to create a delicate blend, which offered wide appeal to the attendees.

Around 300 delegates from dental practices across the UK were greeted by Ben Flewett, Managing Director at Software of Excellence, a Henry Schein One brand, who launched the event by reminding dentists of their preventive responsibilities in the wider healthcare arena and giving some top tips to improve efficiency and boost business performance.

On the first day the event threw open the stage to the keynote, non-dental speakers and delegates gave a deserved standing ovation to motivational speaker and inspirational trainer Steve Head following his thought provoking and highly entertaining talk 'Making the $1 \%$ Difference'. During this session, Steve showed how to create a resilient mindset, with tips on how to manage stress and so improve performance, demonstrating the huge and positive impact that can be made, by employing what might be considered minor changes to one's home and working life.

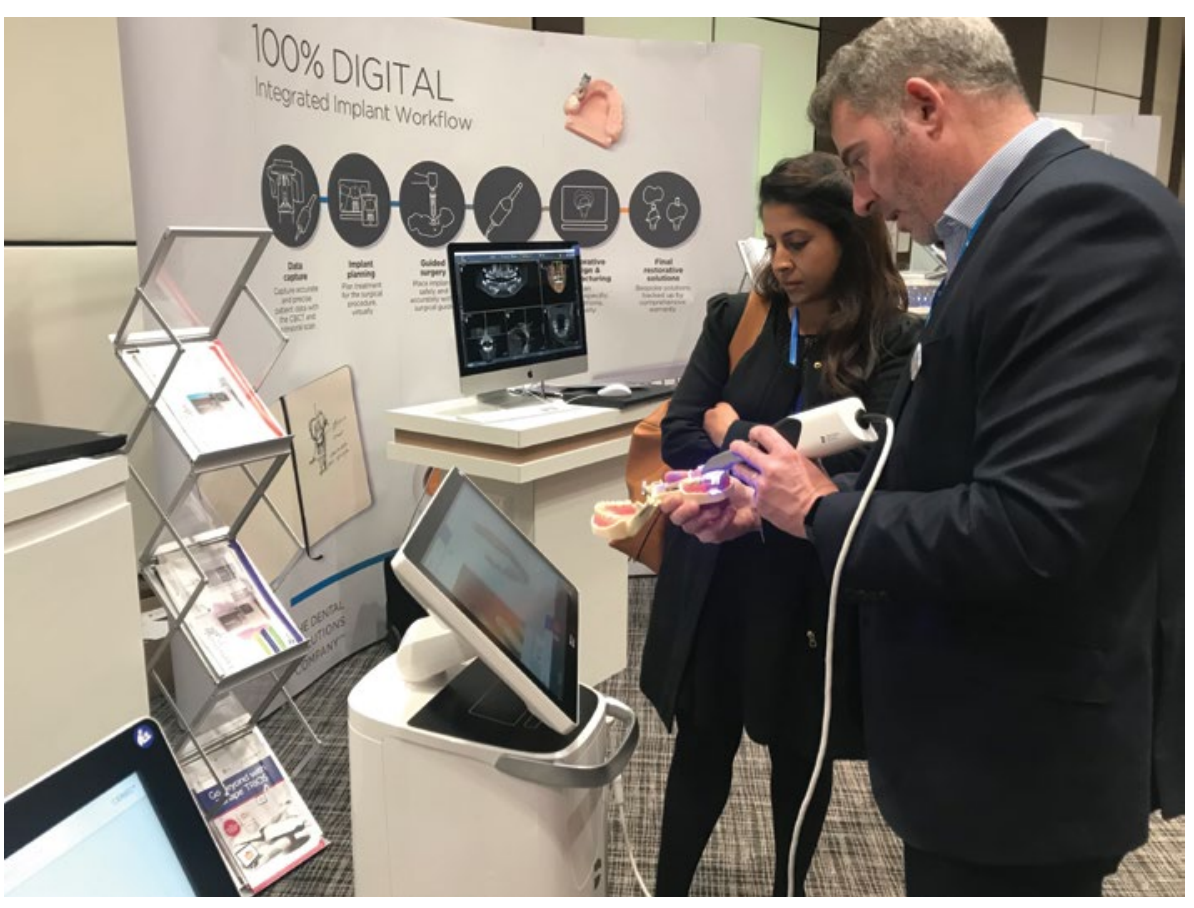

Throughout the two-days a series of smaller presentations and breakout sessions, covering a wide range of topics including digital dentistry, practice performance, teamwork, dental implants, orthodontics, lasers, marketing and finance, meant delegates could choose the topics most relevant to their needs. These sessions built on what had gone before and provided practical tools and perceptive insight into the many different components that go to create a successful, efficient, patient-centred practice.

One of the stars of the Dental Innovation Symposium was undoubtedly Primescan (pictured), the new intraoral scanner from Dentsply Sirona that has opened up a new chapter in digital dentistry. Dr Julian Caplan demonstrated Primescan's speed and accuracy and showcased the range of benefits it brings to numerous clinical situations.

With over 20 exhibitors, including 3Shape, Formlabs and Ivoclar Vivadent as well as Henry Schein companies Software of Excellence, MediEstates, and BioHorizons, the exhibition arena was a hive of activity for the whole two-day period.

As dentistry becomes increasingly competitive for dental professionals, the need to differentiate in the minds of patients is crucial. By utilising new technology and following standard business practices, dentists can achieve a smoother, more efficient workflow, provide better clinical outcomes for patients and boost practice performance. The Dental Innovation Symposium ticked all these boxes, and furthermore, provided delegates with practical advice and tools to help their practices grow. 